\title{
Simultaneous Pre-concentration and Micro-extraction of Organophosphorus Residues Using Magnetic Nanoparticles and Measuring it by Gas Chromatography Device with Flame Ionization Detector
}

\author{
Diyanoosh Mardanbeigi and Mahmoud Ebrahimi* \\ Department of Chemistry, Mashhad Branch, Islamic Azad University Mashhad, Iran. \\ DOI: http://dx.doi.org/10.13005/bbra/1903
}

(Received: 11 June 2015; accepted: 11 August 2015)

\begin{abstract}
At the present study, solid phase micro extraction based on nano-magnetite has been studied as a rapid, sensitive and cost effective method through using iron magnetic nanoparticles. The reason for using iron magnetic nanoparticles is because of easy and rapid controlling and isolating the particles using magnetic field . In technique of solid phase micro extraction based on magnetite nanoparticles (MNs). Magnetic nanoparticles have been applied through direct method for extracting and measuring of the organophosphorus pesticides (OPs); diazinon, fenitrothion and malathion from aqueous samples at the same time through using gas chromatography with flame ionization detector. Firstly, magnetic nanoparticles were synthesized from $\mathrm{Fe}^{2+} / \mathrm{Fe}^{3+}$ salts using chemical coprecipitation method. Magnetic nanoparticles have been also applied directly for purpose of simultaneous extraction of OPs. Effect of some factors such as $\mathrm{pH}$, aqueous phase volume, stirring rate of extraction and desorption and time of extraction have been investigated. Under optimal conditions, concentration factor was also obtained to 1344 , 1257 and 1681 at diazinon, malathion and fenitrothion respectively. The proposed method has been applied for purpose of simultaneous pre-concentration and measurement of OPs in aqueous samples is satisfactorily.
\end{abstract}

Key words: Organophosphorus, hollow fiber, microextraction, graphene oxide.

Solid phase microextraction (SPME) was presented by Politian in early 1990s and has been already applied mostly for preparation of sample ${ }^{1}$. Pesticides are substances intended for preventing, destroying, repelling or mitigating any pest. They are widely used on a variety of crops in the current agricultural practices, and the residues are found in foods ${ }^{2}$. However, the majority of pesticides demonstrate high acute toxicity to human beings $^{2,3}$. Therefore, it is necessary to develop analytical methods to survey OPs residue in

\footnotetext{
* To whom all correspondence should be addressed.
}

aqueous and biological samples, fruits and vegetables for evaluation of the food safety and possible risks to human health.

Recently, great attention has been paid to the synthesis of tha nanoparticles like spheres, nanotubes, nanocages and nanohorns using different materials. Among them, magnetic nanoparticles have attracted broad attention due to their potential applications in magnetic resonance imaging ${ }^{1}$, drug delivery ${ }^{2}$ hyperthermia treatment $^{3}$ and analytical applications ${ }^{4-6}$. As for sample treatment proce- dures, superparamagnetic nanoparticles represent one of the most exciting prospects in analytical nanotechnology since they can be easily isolated from the matrix by using an external magnetic field without retaining residual 
magnetization after its removal. The main advantages over traditional solid-phase extraction sor- bents rely on large surface areas, high extraction efficiencies, use of reduced amounts of sample and of toxic organic solvents. Different magnetic oxides have been synthesized using coprecipitation method $^{7}$, water-in-oil microemulsions ${ }^{8}$, hydrothermal and solvothermal synthesis techniques ${ }^{9-11}$. Magnetite $\left(\mathrm{Fe}_{3} \mathrm{O}_{4}\right)$ is the best-suited magnetic oxide for the above-cited applications, since it was widely characterized in the past and shows ferrimagnetic ordering at room temperature with a saturation magnetization of 92 $\mathrm{emu} / \mathrm{g}^{12}$. Taking into account that electrical, optical and mag- netic properties of nanoparticles strongly depend on their size, the synthesis of monodisperse nanoparticles with controlled particle sizes is of key importance ${ }^{13-15}$.

The method can provide accuracy results with trace concentration of analytes and can also prevent the loss and wastage during extraction, concentration and purification in different traditional method.

Today, with the increasing development of nanotechnology in different fields, the need for more accurate analysis methods has been increased and different analysis methods in scope of nanotechnology are being widely applied. Using nanoparticles in solid phase micro extraction is practically based on dividing the analyte between the sample and static phase covered on the solid surface. The type of applied coverage has basic role in output of isolation and analysis. Structured nanoparticles can be useful instruments for this method because of their absorption capacity ${ }^{2}$.

Agricultural advances in developed countries have been to high extent depended on discovering and introducing different types of pesticides $^{7}$, which have important role in controlling pesticides and protecting vegetation. However, increase in production of pesticides for agricultural and healthcare uses has caused pollution of air and water and has also created some dangers for the environment and health of species.

In order to determine remains of pesticides, different methods can be applied. Most of the methods are based on extraction, concentration and chromatography. Diazinon has been supplied to the market with brands such as
Neocidol, Sarulex and Diazol.

Diazinon has been supplied to the market with brands such as Neocidol, Sarulex and Diazol Analytical chemistry is in close relationship with nanotechnology. The relationship can be interpreted as mutual services of the two sciences to each other. although the studies on them are being developed increasingly. At the present study, magnetic nanoparticles have been applied for purpose of conducting simultaneous micro-extraction of Malathion, fenitrothion and diazinon. Then, components of mixture have been analyzed based on their different migrations along the gas chromatography column.

\section{EXPERIMENTAL}

\section{Apparatus}

The Varian 3800CP gas chromatography (Palo Alto, CA, USA) equipped with a flame ionization detector was employed for determination of the analytes. A DB-5 (5\% biphenyl $+95 \%$ po ly dimethyl siloxane) fused-silica capillary column (30m $\times 0.25 \mathrm{~mm}$ i.d. and $0.25 \mu \mathrm{m}$ film thickness) was applied for separation of the analytes. The GC split valve was opened (split ratio: 1/20) and nitrogen was used as a carrier gas at the constant flow rate of $1.5 \mathrm{~mL} / \mathrm{min}$. The column oven was initially held at 50 æ\% for $3 \mathrm{~min}$, programmed to $150 æ \% \mathrm{C}$ at a rate of $12 æ \% \mathrm{C} / \mathrm{min}$ and then to $240 æ \% \mathrm{C}$ at $20 æ \% \mathrm{C} /$ min. The injections were carried out using a $10 \mu \mathrm{L}$ Hamilton microsyringe (Bonaduz, Switzerland) and $10 \mathrm{~mL}$ extraction vial. Stirring of the solutions was carried out by a Heidolph MR3001magnetic stirrer (Schwabach, Germany) and a $8 \times 1.5 \mathrm{~mm}$ magnetic stirring bar.

Shaker device made by Pars Azma Company. ethanol ,methanol (HPLC grade) $\mathrm{FeCl}_{3} \cdot 6 \mathrm{H}_{2} \mathrm{O}, \mathrm{FeCl}_{2} \cdot 4 \mathrm{H}_{2} \mathrm{O} ; 37 \%$ hydrochloric acid; $32 \%$ ammoniac-; $65 \%$ nitric acid were purchased from Merck. Diazinon, fenitrothion and malation were purchased from China High Hope Fortone Ag.Co with.

\section{Synthesis of magnetic nanoparticles}

The magnetic $\mathrm{Fe}_{3} \mathrm{O}_{4}$ NPs were prepared by the chemical co-precipitation method. Briefly, $5.2 \mathrm{~g}$ of $\mathrm{FeCl} 3-6 \mathrm{H} 2 \mathrm{O}, 2.5 \mathrm{~g} \mathrm{FeCl}_{2}-4 \mathrm{H}_{2} \mathrm{O}$, and $0.85 \mathrm{~mL}$ $\mathrm{HCl}$ (conc.) were dissolved in $25 \mathrm{~mL}$ water under a $\mathrm{N}_{2}$ stream. This solution was added drop-wise into $250 \mathrm{~mL}$ of sodium hydroxide under 
a nitrogen atmosphere and stirred for $30 \mathrm{~min}$. The resulting black precipitate was separated with a magnet and washed several times with degassed water; it was stored in $100 \mathrm{~mL}$ degassed water under a nitrogen atmosphere ${ }^{7}$. it was cooled under ambient temperature. Synthesized nanoparticles were absorbed by magnetic field.

\section{Magnetic solid phase extraction (MSPE) Procedure}

for each solution $10 \mathrm{mg}$ MNPs was added to $5 \mathrm{~mL}$ of water sample containing OPs in a $10 \mathrm{~mL}$ vial and stirred for 20 min with speed of $300 \mathrm{rpm}$. After performing the extraction at room temperature, the magnetic sorbents were collected using an external magnet, and the supernatant water was decanted. The extracted analytes was desorbed using $0.1 \mathrm{~mL}$ of methanol and subsequently $1 \mu \mathrm{L}$ aliquot was injected into the GC-FID system for analysis. For each experiment, four replicate extractions were performed.

\section{RESULTSAND DISCUSSION}

\section{Characterization of Functionalized Magnetic nanoparticles}

Particle interactions with their environment are greatly affected by their surface functionality. the characterization was performed by IR spectroscopy. The FT-IR spectra for $\mathrm{Fe}_{3} \mathrm{O}_{4}$ MNPs is shown in Fig. 4. The broad feature in the range $3,440-3,225 \mathrm{~cm}^{-1}$ was due to $-\mathrm{H}$ stretch (m1), which corresponds to the hydroxyl group attached by the hydrogen bonds to the iron oxide surface, and also the water molecules chemically adsorbed to the magnetic nanoparticl surface ${ }^{16}$. The transmittance waveband from 635 to $573 \mathrm{~cm}^{-1}$, which corresponds to the metal-oxygen bonds, is considered as an indication of the ferrite formation ${ }^{17}$. Figure 4 shows the particle morphology of the prepared MNPs by transmission

Table1. Figures of merit of the proposed method in the determination of the organophosphorus pesticides in aqueous samples

\begin{tabular}{lccccc}
\hline Analtye & DLR $(\mathrm{mg} / \mathrm{L})$ & Regression equation & $\mathrm{R}^{2}$ & LOD $(\mathrm{mg} / \mathrm{L})$ & \%RSD (n=5) \\
\hline Diazinon & $0.002-20$ & $\mathrm{Y}=21764.35+14736 \mathrm{C}$ & 0984 & 0.00081 & 4.4 \\
Fenitrothion & $0.0008-15$ & $\mathrm{Y}=19574.8+11367.6 \mathrm{C}$ & 0.9935 & 0.0007 & 4.6 \\
Malathion & $0.0005-20$ & $\mathrm{Y}=16734.74+4365 \mathrm{C}$ & 0.9945 & 0.0004 & 3.9 \\
\hline
\end{tabular}

Table 2. Comparison of some methods which were used for determination of pesticides compounds

\begin{tabular}{llllllll}
\hline No & Date & Matrices & ExtractionTechnique & LOD & R & RSD\% & Refence \\
\hline 1 & 2004 & Honey & SPME & $0.08-20 \mathrm{mg} \mathrm{kg}^{-1}$ & 0.996 & $3.6-7.6$ & {$[22]$} \\
2 & 2004 & Herbal infusions & SPME & $0.13-1.1 \mathrm{mg} \mathrm{mL}^{-1}$ & 0.974 & $1.3-12.1$ & {$[23]$} \\
3 & 2004 & Food & SPME & $0.01-0.1 \mathrm{ng} \mathrm{gr}^{-1}$ & 0.998 & $2.1-12.1$ & {$[24]$} \\
4 & 2007 & beverage & LPME & $0.1-1.7 \mu \mathrm{L} \mathrm{L}^{-1}$ & 0.95 & $6.1-11.5$ & {$[25]$} \\
5 & 2008 & Water & SPME & $0.17-0.29 \mu \mathrm{L} \mathrm{L}^{-1}$ & 0.998 & $2 .-2.7$ & {$[26]$} \\
6 & 2008 & Water & LPME & $0.01-0.04 \mathrm{mg} \mathrm{L}^{-1}$ & 0.999 & - & {$[27]$} \\
7 & 2010 & Water & SPME & $0.1-1 \mathrm{pg} \mathrm{mL}^{-1}$ & 0.996 & $2-10$ & {$[28]$} \\
\hline
\end{tabular}

Table 3. Detected concentrations (mg/L) of organophosphorus pesticides in surface water (Mashhad, Iran) and river water

\begin{tabular}{|c|c|c|c|c|c|c|}
\hline \multirow[t]{2}{*}{ Analtye } & \multicolumn{2}{|c|}{ Concentration (mg/L ) } & \multicolumn{2}{|c|}{ RSD\% $(n=5) \%$} & \multicolumn{2}{|c|}{ Relative recovery } \\
\hline & surface water & river water & surface water & river water & surface water & river water \\
\hline Diazinon & 1.98 & 1.84 & 2.6 & 3.6 & 91 & 92 \\
\hline Fenitrothion & 1.8 & 1.8 & 3.6 & 4.7 & 90.5 & 90 \\
\hline Malathion & 1.86 & 1.78 & 4.3 & 4.2 & 93 & 89.8 \\
\hline
\end{tabular}

e Relative recovery after spiked $2 \mathrm{mg} / \mathrm{l}$ of analytes 
<smiles>CCOC(=O)CC(SP(=S)(OC)OC)C(=O)OCC</smiles>

Fig. 1.<smiles>CCOP(=S)(OCC)Oc1cc(C)nc(C(C)C)n1</smiles>

Fig. 1.<smiles>CCOC(=O)CC(SP(=S)(OC)OC)C(=O)OCC</smiles>

Fig. 3.

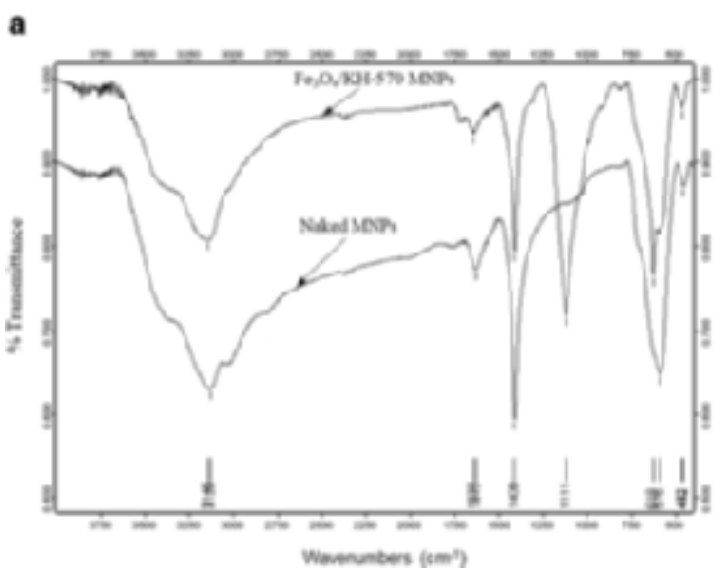

electron microscopy (TEM). It is seen that the particles are well dispersed with particle size about 20-40 nm. After ensure of success of the method, relevant examinations of optimization of micro extraction conditions were conducted. Various factors can affect amount of micro extraction and pre-concentration. In order to enhance efficiency of microextraction, the conditions should be optimized. Optimized factors in this phase include extraction time, desorption time, capacity of donor phase and mixing speed.

\section{Optimization}

Effect of type desorption solvent

Selecting organic solvent is one of the most important effective factors in extraction process and proper selection of it can result in good sampling. Offline desorption performed using minimum volume of desorption solvent required to completely immerse the SPME fiber segment. Different desorption solvents such as, cyclohexane, acetonitrile, methanol and 1-octanol were employed. Based on the obtained results, methanol was found to get the best extraction efficiency, while its chromatographic peak was easily separated from the analyte peaks (see Fig. 5). Meanwhile, the extract was stable at the extraction period due to its low vapor pressure at the extraction conditions. Therefore, methanol was selected as the desorption solvent.

Pesticide compounds at the concentration level of $1 \mathrm{mg} / \mathrm{L}$, were used in the extraction studies Effect of pH effect

The $\mathrm{pH}$ value of aqueous feed-phase plays an essential role in the extraction process. Considering the feed solution $\mathrm{pH}$ is also one of

b

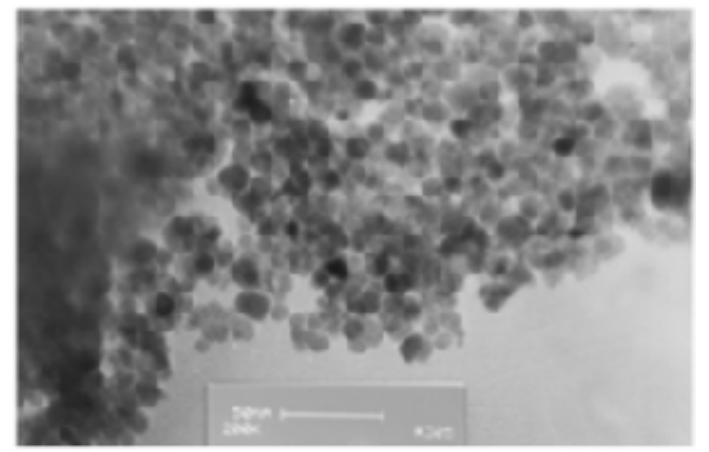

Fig. 4. a) FT-IR spectra of the MNPs, b) TEM image of the magnetic nanoparticles 


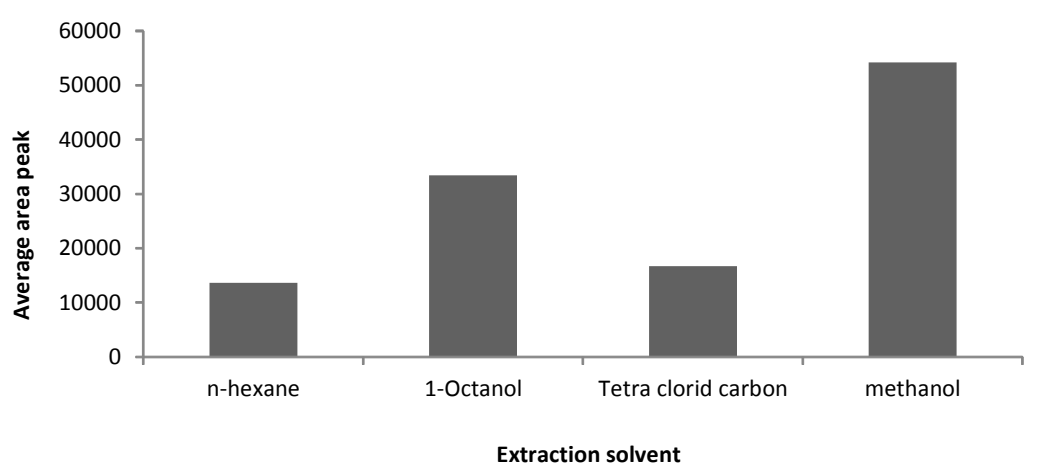

Fig. 5.

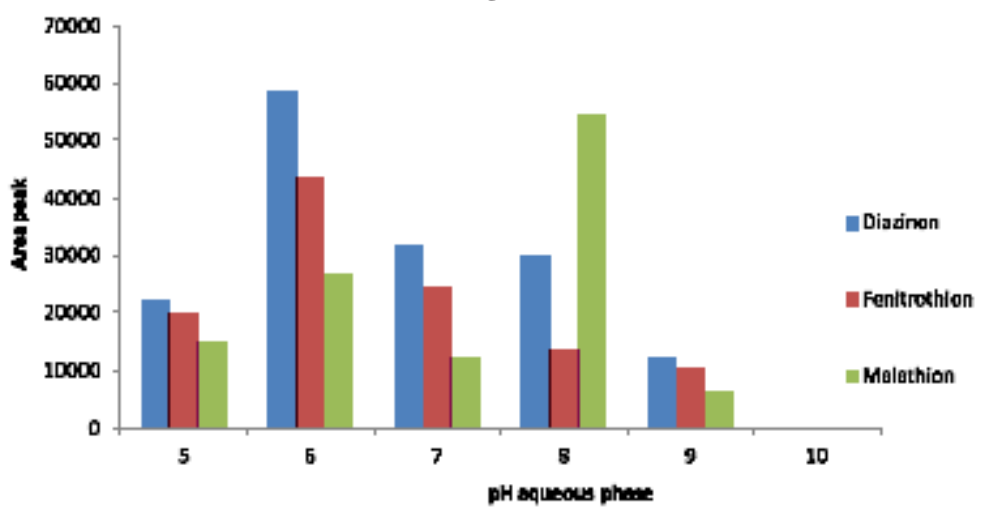

Fig. 6.

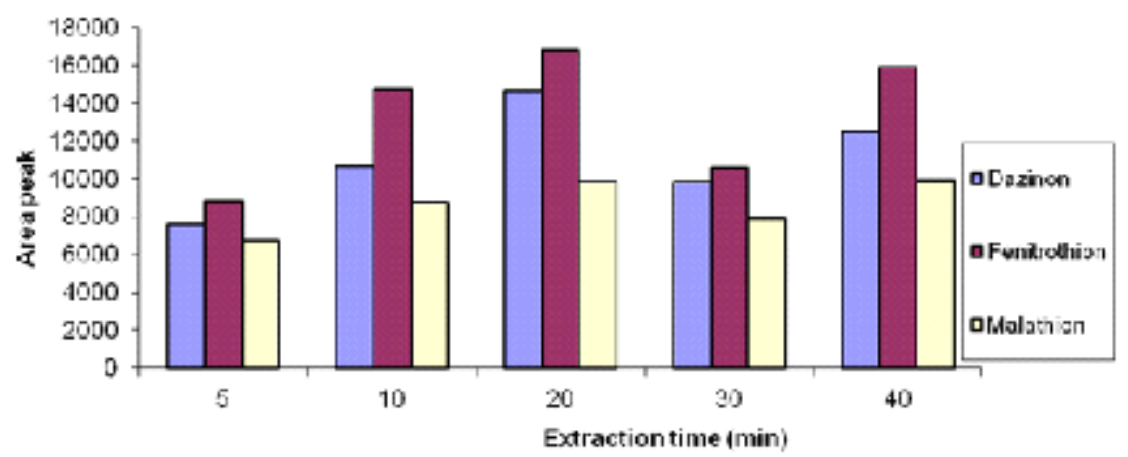

Fig. 7.

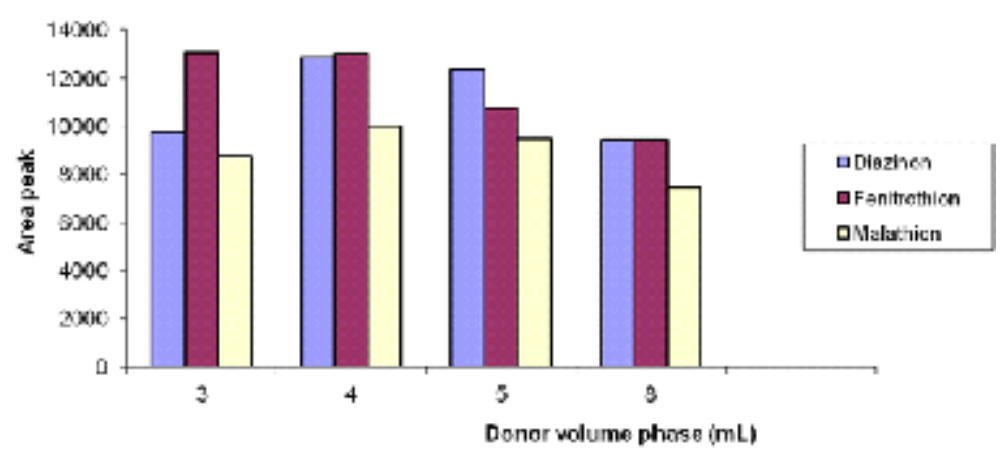

Fig. 8. 
the important factors that it progresses the transfer of OPs from the feed to the adsorbent. Therefore, after survey of the $\mathrm{pH}$ effect in the $\mathrm{pH}$ range $5-11$, by adding the appropriate hydrocholoric acid or sodium hydroxide solution to the aqueous donor phase.

The results confirmed that the analytes extraction performance reached a better level at pH 6 (see Fig. 6). When pH rose above 6, the peak areas of malathion and diazinon decreased rapidly. Afterwards the peak areas were decreased with increasing of $\mathrm{pH}$. It is due to the occurrence of degradation under high alkaline condition. Based on thorough consideration, $\mathrm{pH} 6$ was selected for furtherexperiments.

One of the most important factors in process of surface absorption of malathion, fenitrothion and diazinon on magnetic nanoparticles and finally, increase in efficiency of extraction is $\mathrm{pH}$ level. $\mathrm{pH}$ of solution plays an important role in mechanism of surface absorption of the desired compound on surface of magnetic nanoparticle and form and load of the desired sample. Surface lead of magnetic nanoparticles is condition of $\mathrm{pH}<7$ is positive. On the other hand, compounds of diazinon and malathion were decomposed in strong acid and alkaline $\mathrm{pH}$ ratios. In this study, $\mathrm{pH}$ levels of 10, 9, 8, 7, 6, 5 and 4 have been investigated and it was observed that in $\mathrm{pH}=6$, the maximum level of sub-peak can be obtained. Therefore, $\mathrm{pH}=6$ has been considered here as optimized pH level. In $\mathrm{pH}=6$, level of nanoparticle is positive, molecular form of analytes is stable and the condition is provided for surface absorption that has high extraction efficiency.

The affection of the time on the extraction efficiency is an equilibrium process and extraction time influences on the equilibrium conditions. Over the extraction solute molecules have sufficient chance extraction device and for collection in it. Therefore, extraction time is a significant factor that influences on the extraction efficiency. Extraction was performed from 5 to $60 \mathrm{~min}$ to determine the effect of extraction time on the method efficiency. The results that were shown in Fig. 7 . All the analytes demonstrated that average peak of analytes the highest increase in the peak areas in the period of $20 \mathrm{~min}$. Afterwards the average peak areas were decreased with increasing of extraction time.
So, a period of 30 min was used for the subsequent experiments.

\section{Effect of the stirring rate}

Stirring enhances mass transfer and reduces the time required to reach equilibrium. The instrument's response was recorded for several stirring rates ranging from 100 to $700 \mathrm{rpm}$ for a extraction time of 20 min of $4 \mathrm{~mL}$ aqueous samples with each target analyte concentration of $10 \mu \mathrm{g} /$ $\mathrm{mL}$. The results confirmed that agitation of the sample enhances extraction. However, violent stirring (>300 rpm) resulted in massive air bubble and decreased the preconcentration factors. Therefore, a $300 \mathrm{rpm}$ setting was selected for extraction at the subsequent experiments. On the other hand, it is understood that agitation of the sample enhances desorption.

The effect of this variable on the desorption step was assessed by varying the stirring rate from $100 \mathrm{rpm}$ to $500 \mathrm{rpm}$ using a magnetic stirrer. It was observed that the peak areas of the analytes areincreased with the increase of stirring rate up to $400 \mathrm{rpm}$. Above this value the amount of extracted analyte remained unchanged. Therefore, $400 \mathrm{rpm}$ was set as the optimized rate for subsequent experiments.

\section{Effect of the donor phase volume}

As the analytes are extracted from relatively large sample volumes into a very small volume of acceptor phase, most extraction applications provide substantial analyte efficiency extraction.

As the volume of the sample increases, the efficiency extraction also increases ${ }^{18-20}$. In SPME, extraction is in equilibration and therefore, the amount of analyte partitioning into the acceptor phase becomes independent of the sample volume when this volume is much higher than the product of the partition constant and the volume of the acceptor phase. This implies that increasing the efficiency extraction requires donor phase volume to be large On the other hand, a larger sample volume has disadvantageous to poorer masstransfer kinetics, resulting in worse extraction efficiency [21]. In the present work, the phase donor volume solutions was optimized by changing the volume of the donor phase between 3 and $8 \mathrm{~mL}$ while the amount of magnetic nanoparticles was kept constant. Generally, the extraction efficiency can be improved by increasing the volume of 
donor donor phase. As seen in Fig. 8, however, the extraction results obtained for the analytes were favorable at $4 \mathrm{~mL}$ donor phase volume. Repeatability was decreased in the aqueous donor phase volumes less than $4 \mathrm{~mL}$. While with an increase in the aqueous donor phase volume. This would lead to a decrease in the extraction efficiency. Therefore, we selected a volume of 4.0 $\mathrm{mL}$ as the optimized donor phase volume.

\section{Effect of desorption solvent volume}

In extraction procedure, analytes were desorbed using the appropriate organic solvent system. As this research has shown, methanol is often the best desorption solvent. Desorption solvent volume is important on the desorption capacity of extraction device as well as the overall time required by extraction to reach equilibrium. Four different desorber volumes (0.1-1.0 mL) for spiked target analytes $(10 \mu \mathrm{g} / \mathrm{L})$ were studied. Highest extraction efficiency was observed when $0.1 \mathrm{~mL}$ desorption solvent volume was used. Decrease in peak area response was noted when larger desorption solvent volume applied in the extraction. This phenomenon might be due to the dilution analyteseffects. Repeatability was decreased in the desorption solvent volume less than $0.1 \mathrm{~mL}$. Thus $0.1 \mathrm{~mL}$ was used as the optimal volume desorption solvent.

\section{Evaluation of the method performance Figures of merit}

To evaluate the practical applicability of SPME technique, the figures of merit of this method including correlation coefficient ( $R$ ), the corresponding regression equation, experimental limit of detection (LOD) and linear dynamic range (LDR) were investigated under the best conditions. Calibration curves in aqueous and were plotted against the concentration levels of the pesticides compounds. At each level, four replicate extractions were performed. The results are showed in Table 1.

The method was compared with the other works (Table 2). In comparison with the other conventional sample preparation method, the developed method has the figure of merits of considerable analysis speed, good separation efficiency and improved pre-concentration, high sensitivity and notable precision.

\section{Analysis of Real Samples}

Applicability and reliability of the extraction method to extract the Ops from surface water (Mashhad, Iran) and river water (Akhlamad River, Mashhad, Iran) were investigated.

The experimental results indicated that no analytes were detected in real samples.

Therefore, the method was evaluated by analysis of the samples spiked with known amount of

OPs. The obtained results showed which indicates that the proposed method is repeatable. The analytical results of real samples are given in Table 3.

Detected concentrations (mg/L) of organophosphorus pesticides in surface water (Mashhad, Iran) and river water

\section{CONCLUSION}

In this study, magnetic adsorbents synthesized and used as a SPME nano-adsorbent to separate and pre-concentrate trace amount of diazinon, malathion and fenitrothion prior to GCFID analysis in aqueous environmental samples.

The high extraction efficiency of MNPs adsorbents was caused by their large surface areas and strong adsorption ability of magnetic adsorbent. The MNPs adsorbents show a large surface area, and satisfactory extraction for analytes was obtained in analysis of real samples which indicate good selectivity of the adsorbents and high recoveries (see table 3 ). The proposed method can be applied for another pesticides and in legal medicine centers for analysis of biological samples such as human hair samples to identify the causes of drugs intoxication and death. This method was also applicable for environmental pollution control for analysis of aqueous environmental samples such as river and surface water samples.

\section{REFERENCES}

1. M.A. Sogorb, E. Villanova, Toxicol. Lett.2002; 128: 215

2. F.M. Raushel, Curr. Opin.Microbiol. 2002; 5: 288

3. M. Valcaercel, S. Caerdenas, M. Gallego, Trend anal chem, 1999; 18: 685

4. D. A. Wells, High throughput bioanalytical sample preparation, Methods and automation Strategies vol. 5, Elsevier B.V., Amsterdam, 2003

$5 \quad$ H. Kataoka, Trend Anal Chem, 2003; 224: 232.

6. James D.ingles. JR, Stanley R. Crouch. Spectro 
chemical Analysis, Prentic-Hall, Inc, New Jersey, 1988

7. D.Das, U.Gupta, Arabinda K. Dasm, Trend Anal Chem, 2012; 38: 163.

8. J. D. WINEFORDNER,Sample Preparation Techniquesin Analytical Chemistry , John Wiley \& Sons, Inc., Hoboken, New Jersey, 2003.

9. R.E.majors, Trends in Sample preparation for Chromatography, LC/GC Magazine,Agilent Technologies, 2002 .

10. Z. Xiong, Z. Ji, Xiaolin Wu, Y. Chen, H. Chen, Fuel 2008; 87: 3096.

11. J. Pawliszyn, Comprehensive Sampling and Sample Preparation, Elsevier Science and Technology, 2012.

12. S.Saeed .Albaseer, R.NageswaraRao, Y.V.Swamy, K.Mukkanti , J ChromatogrA, 2010; 1217: 5537

13. C. Wagner, K. H. Esbensen, Renewable and Sustainable Energy Reviews, 2012; 16: 504

14. N.C.Van de Merbel,H.Linheman, JChromatogr A, 1999; 725: 13 .

15. C.E. Meloan, Chemical Separations, Principles, Techniques, and Expriments,Wiley, New York 1999.

16. Creanga D, Calugaru G, Physical investigations of a ferrofluid based on hydrocarbons. J Magn Magn Mater 2005; 289: 81-83. doi:10. 1016/ j.jmmm.2004.11.023

17. Mashhadizadeh MH, Karami Z., Solid phase extraction of trace amounts of $\mathrm{Ag}, \mathrm{Cd}, \mathrm{Cu}$, and $\mathrm{Zn}$ in environmental samples using magnetic nanoparticles coated by 3-(trimethoxysilyl)-1propantiol and modified with 2-amino-5mercapto-1,3,4-thiadiazole and their determination by ICP-OES. J Hazard Mater 2011; 190:1023-1029. doi:10.1016/j.jhazmat. 2011.04.051

18. A. Sarafraz-Yazdi, Z. Es'haghi, J. Chromatogr. A 2005; 1082: 136.

19. A. Sarafraz-Yazdi, Z. Es'haghi, Chromatographia 2006; 63: 563.

20. E. Psillakis, N. Kalogerakis, Trends Anal. Chem. 2003; 22: 565.

21. Z. Es’haghi, Anal. Chim. Acta 2009; 641: 83.

22. Y. Liu, M. Wang, J. Li, Z.Y. Li, P. He, H.T. Liu, J.H. Li, Chem. Commun. 2005; 13: 1778.

23. C. Blasco, M. Fernández, Y. Picó, G. Font, J. Chromatogr. 2004; 1030: 77.

24. V.G. Zuin, A.L. Lopes, J.H. Yariwake, F. Augusto, J. Chromatogr. A 2004; 1056: 21.

25. M. Riu, M. Mestres, O. Busto, J. Guasch, J. Chromatogr. A 2006; 1107: 240.

26. Q. Zhou, H. Bai, G. Xie, J. Xiao, J. Chromatogr. A 2009; 1188:148.

27. M. Fernandez-Alvarez, M. Llompart, J.P. Lamas, M. Lores, C. Garcia-Jares, R. Cela, T. Dagnac, J. Chromatogr. A 2008; 1188: 154.

28. H. Bagheri, Z. Ayazi, E. Babanezhad, Microchem. J. 2010; 94: 1. 